\title{
Sustained Splits of Attention within versus across Visual Hemifields Produce Distinct Spatial Gain Profiles
}

\author{
Sabrina Walter ${ }^{1}$, Christian Keitel ${ }^{2}$, and Matthias M. Müller ${ }^{1}$
}

\begin{abstract}
Visual attention can be focused concurrently on two stimuli at noncontiguous locations while intermediate stimuli remain ignored. Nevertheless, behavioral performance in multifocal attention tasks falters when attended stimuli fall within one visual hemifield as opposed to when they are distributed across left and right hemifields. This "different-hemifield advantage" has been ascribed to largely independent processing capacities of each cerebral hemisphere in early visual cortices. Here, we investigated how this advantage influences the sustained division of spatial attention. We presented six isoeccentric lightemitting diodes (LEDs) in the lower visual field, each flickering at a different frequency. Participants attended to two LEDs that were spatially separated by an intermediate LED and responded to synchronous events at to-be-attended LEDs. Task-relevant pairs of LEDs were either located in the same hemifield ("within-
\end{abstract}

hemifield" conditions) or separated by the vertical meridian ("across-hemifield" conditions). Flicker-driven brain oscillations, steady-state visual evoked potentials (SSVEPs), indexed the allocation of attention to individual LEDs. Both behavioral performance and SSVEPs indicated enhanced processing of attended LED pairs during "across-hemifield" relative to "within-hemifield" conditions. Moreover, SSVEPs demonstrated effective filtering of intermediate stimuli in "across-hemifield" condition only. Thus, despite identical physical distances between LEDs of attended pairs, the spatial profiles of gain effects differed profoundly between "across-hemifield" and "within-hemifield" conditions. These findings corroborate that early cortical visual processing stages rely on hemisphere-specific processing capacities and highlight their limiting role in the concurrent allocation of visual attention to multiple locations.

\section{INTRODUCTION}

Behaviorally relevant stimuli have to be prioritized constantly over irrelevant sensory input because the visual system possesses limited processing capacity (Kahneman, 1973). Neural mechanisms of attention that selectively enhance relevant and suppress irrelevant stimuli guide this prioritization (Kastner \& Ungerleider, 2001). Visuospatial attention, in particular, has been conceptualized as a unitary spotlight (Posner, Snyder, \& Davidson, 1980) or zoom lens (Eriksen \& St. James, 1986) that can be cast selectively on locations within the visual field. Crucially, stimuli that fall within the spotlight receive a processing advantage over stimuli that fall outside. Over the years, a number of studies provided empirical evidence that attention cannot only be allocated in space quite flexibly (Müller \& Hübner, 2002; Awh \& Pashler, 2000 ) but that it can also be deployed to two locations that are separated by an intermediate distractor position, suggesting that the spotlight of attention can be split into two noncontiguous spotlights (Müller, Malinowski, Gruber, \& Hillyard, 2003).

Despite empirical evidence from behavioral (Awh \& Pashler, 2000) and electrophysiological (Malinowski, Fuchs, \& Müller, 2007; Müller et al., 2003) MRI and fMRI

\footnotetext{
${ }^{1}$ University of Leipzig, ${ }^{2}$ University of Glasgow

studies (McMains \& Somers, 2004, 2005) in an extensive review of the literature regarding visuospatial attention to spatially separated locations, Jans et al. came to the conclusion that "the jury is still out" (Jans, Peters, \& De Weerd, 2010). In other words, even after many years of research "splitting the spotlight" is still an interesting topic in cognitive (neuro-)science and triggers controversy. To date, it is still an open issue whether successful splits require attended locations to be placed in different visual hemifields. This question has emerged from the finding that attention effects are most pronounced when two attended stimuli were positioned in the left and right hemifields, respectively (McMains \& Somers, 2004, 2005; Müller et al., 2003). Given that splitting the spotlight appears more difficult or even impaired when two to-be-attended and an intermediate to-be-ignored position fall within one hemifield (Kraft, Kehrer, Hagendorf, \& Brandt, 2011; Malinowski et al., 2007; McMains \& Somers, 2004), this phenomenon seemingly depends on constraints during concurrent stimulus processing within one hemisphere.

A parallel line of research consistently reported increased behavioral performance during various multifocal attention tasks when stimuli were distributed across left and right visual hemifields as compared with when stimuli concentrated within one hemifield (Alvarez, Gill, \& Cavanagh, 2012; Harasawa \& Shioiri, 2011; Kraft et al., 2005, 2007; Alvarez \& Cavanagh, 2005; Awh \& 
Pashler, 2000). Sereno and Kosslyn (1991) referred to this behavioral benefit as the different-hemifield advantage. They suggested that each hemisphere is able to perform at least some aspects of the task independently because hemispheres relied on "independent resource pools."

Recently, Franconeri, Alvarez, and Cavanagh (2013) have extended this idea by replacing the relatively vague concept of resource pools with so-called competitive content maps. These maps depict cortical representations of external space on which stimuli compete for processing. Spatiotopic maps found throughout most sensory and motor cortices (Purves et al., 2004) support this notion. Importantly, the boundary between left and right visual hemifields decreases interitem competition at early processing stages during across-hemifield performance because each item is represented by a separate map in a different cortical hemisphere. Conversely, competition is high when attended stimuli fall within the same hemifield and, thus, within one map in one hemisphere (Franconeri et al., 2013).

Despite differences in the conceptualization (i.e., resource pools vs. content maps), both models come to the same conclusion, namely, greater competition in within- compared with across-hemifield (or hemisphere) stimulus configurations, resulting in the effect that the spotlight of attention splits more readily across than within hemifields.

In the present experiment, we measured cortical facilitation in early visual areas of the human brain during sustained spatial attention. Participants viewed six lightemitting diodes (LEDs), aligned on a semicircle in the lower visual field. A cue at the beginning of each trial indicated to-be-attended task-relevant LED positions while irrelevant positions had to be ignored. Participants were asked to perform a detection task at covertly attended LED pairs. Paired LED positions were always separated by an intermediate LED. Hence, to perform the task, participants had to split their spotlight of attention between two LEDs either located "within" or "across" visual hemifields.

For several seconds, each LED flickered at a unique frequency, thereby eliciting a distinct brain response in the simultaneously measured EEG, the steady-state visual evoked potential (SSVEP; Regan, 1989). Cortical currents giving rise to SSVEPs have been found to originate in early visual cortices (Quigley, Andersen, \& Müller, 2012; Fuchs, Andersen, Gruber, \& Müller, 2008; Di Russo et al., 2007). SSVEPs thus allow for the separation of stimulusspecific brain activity in the EEG and enable conclusions about early visual processing and interactions of multiple concurrently presented elements in the visual field (Keitel, Andersen, Quigley, \& Müller, 2013; Andersen, Müller, \& Hillyard, 2011; Müller et al., 1998). Critically, SSVEP amplitudes can be modulated by attention (Müller, Picton, et al., 1998; Morgan, Hansen, \& Hillyard, 1996) as well as interstimulus competition (Keitel, Andersen, \& Müller, 2010; Fuchs et al., 2008). Previous experiments have exploited these characteristics to study modulations of visual process- ing under conditions of multifocal attention (Itthipuripat, Garcia, \& Serences, 2013; Störmer, Winther, Li, \& Andersen, 2013; Müller et al., 2003).

More recently, SSVEPs have been instrumental in investigating the neural underpinnings of the different-hemifield advantage. In a previous study, we probed hemispheric interactions during sustained unilateral and bilateral attention (Walter, Quigley, \& Müller, 2014). Participants viewed an arrangement of four LEDs, one positioned in each quadrant of the visual field. They performed a luminance discrimination task on cued pairs of LEDs that were aligned horizontally across or vertically within hemifields. In this series of experiments, consistent with the availability of additional processing capacities, SSVEP amplitudes indicated greater attentional gain in "across-hemifield" than "withinhemifield" conditions. These findings corroborated predictions of the different-hemifield advantage account (Sereno \& Kosslyn, 1991) and the competitive content maps account (Franconeri et al., 2013), as discussed above.

In a control condition, this study further revealed that attending to only one LED in one quadrant and attending to two LEDs in "across-hemifield" conditions led to comparable gain effects. Crucially, this was not the case in "within-hemifield" conditions (=two attended LEDs in one hemifield) in which gain effects were significantly reduced. First, this demonstrated that the number of stimuli that were attended simultaneously by one hemisphere influenced their representational strength in visual cortex. Moreover, together with findings from earlier studies on shifts of spatial attention (Müller, Teder-Sälejärvi, \& Hillyard, 1998), this implied that attending to stimuli in one visual hemifield did not influence the processing in the opposite unattended hemifield. Altogether, we reported supportive experimental evidence for hemisphere-specific attentional influences on processing in early visual cortex.

Similar results have been obtained while employing frequency tagging in a motion object tracking (MOT) paradigm (Störmer, Alvarez, \& Cavanagh, 2014). MOT tasks typically require attending to one or more stimuli that traverse the visual field concurrently but on different trajectories (Atmaca et al., 2013; Alvarez \& Cavanagh, 2005). Störmer et al. (2014) adapted the MOT paradigm to allow for a comparison between attentional resource allocation during the tracking of two initially cued stimuli (out of 8; two moving within each quadrant) that were positioned either within or across visual hemifields. The authors reported that attended stimuli received substantial gain effects only during "across-hemifield" conditions even after they reduced object speed in (more difficult) "withinhemifield" conditions to match task performance between conditions. When both stimuli had to be tracked within the same hemifield, gain effects were absent.

Despite these interesting results, it remains to be shown how the different-hemifield advantage influences sensory gain when two simultaneously attended stimuli are placed at noncontiguous locations that are separated by an intermediate irrelevant but possibly interfering third stimulus. 
Taking into account the existence of the differenthemifield advantage for multifocal attention tasks (regardless of its conceptualization, i.e., independent resource pools or content maps), we expected that SSVEP amplitudes elicited by to-be-ignored intermediate LEDs would be significantly smaller compared with those elicited by to-be attended LEDs in "across-hemifield" conditions. If, however, attended LED pairs were located in one visual hemifield, we expected a smaller difference between processing at attended and intermediate ignored positions given the greater competition for processing capacity within a single hemisphere. In addition, superior behavioral performance (Alvarez et al., 2012; Alvarez \& Cavanagh, 2005; Sereno \& Kosslyn, 1991) and greater gain effects for attended stimuli during "across-hemifield" conditions (Störmer et al., 2014; Walter et al., 2014) should indicate the different-hemifield advantage, thus replicating earlier findings.

\section{METHODS}

\section{Participants}

Fifteen volunteers participated in the experiment. Data of one participant had to be excluded from analysis because of contamination of trials by blinks and other artifacts. We excluded another data set because EEG recordings of the respective participant did not show SSVEPs for stimulation frequencies $>11 \mathrm{~Hz}$. Data of the remaining 13 participants entered analyses (10 women; mean age $=22$ years, $S D=3$ years, range $=19-29$ years; all right-handed). All reported normal or corrected-tonormal vision and gave written consent. The study met the requirements of the Declaration of Helsinki as well as of local and national ethics committees. Participants received monetary compensation or course credit.

\section{Stimulus Material}

Participants viewed an arrangement of six red LEDs, sized $2.1^{\circ} \times 1.43^{\circ}$ of visual angle each (height $\times$ width), at a viewing distance of $60 \mathrm{~cm}$. LEDs were aligned on a semicircle in the lower visual field. A central white cross of $1^{\circ} \times$ $1^{\circ}$ of visual angle in the center of the arrangement served for fixation. The center-to-center distance between the fixation cross and surrounding LEDs was $6.2^{\circ}$ of visual angle. Each LED flickered at a unique rate of 9, 10,11, 12, 13 or $14 \mathrm{~Hz}$ with a 50:50 on-off duty cycle. Frequencies were kept within a close range to control for differences in stimulus perception across flicker rates. Even frequencies were used for stimulation in the left hemifield; odd frequencies were used for stimulation in the right hemifield (see Figure 1A).

The overall luminance of the stimulus arrangement was measured when LEDs were constantly "on" and corresponded to $29 \mathrm{~cd} / \mathrm{m}^{2}$. LEDs were controlled by a custommade USB interface that was connected to a PC running the Cogent Graphics toolbox (John Romaya, Laboratory of Neurobiology at the Wellcome Department of Imaging Neuroscience, London, United Kingdom) and customwritten routines in a MATLAB environment (The MathWorks, Natick, MA).

\section{Experimental Paradigm}

Figure 1 provides a schematic overview of the stimulus configuration and experimental design. The experiment

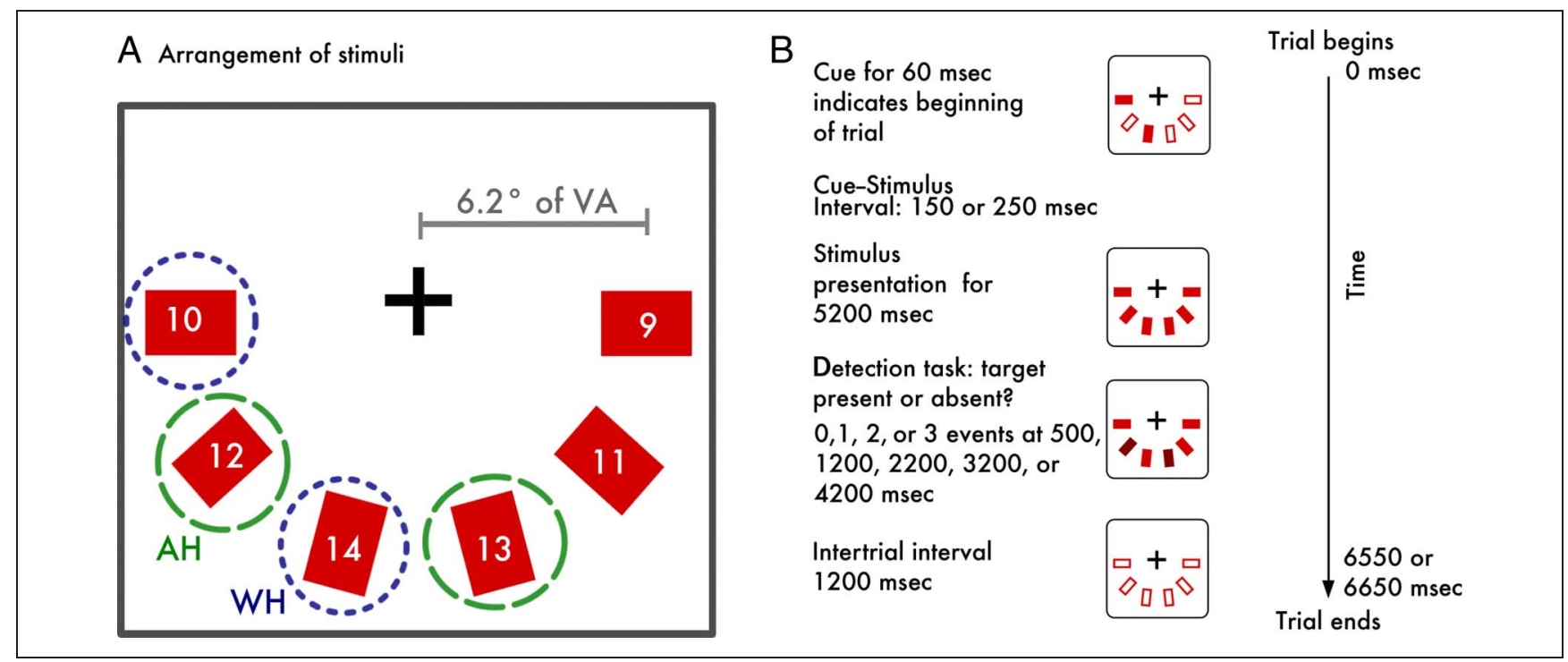

Figure 1. (A) Arrangement of LED stimuli in the lower visual field and schematic overview for split-attention conditions across (AH, green dashed circles) and within (WH, blue dotted circles) hemifields. Numbers indicate flicker frequencies of LEDs in Hertz. Eccentricities of LEDs from central fixation, indicated by the gray scale, corresponded to $6.2^{\circ}$ of visual angle (VA). (B) Experimental paradigm, exemplarily shown for one condition. Participants were instructed to attend the cued LED pair while keeping gaze fixated centrally. 
consisted of four conditions: Participants attended to pairs of LEDs that flickered at 12 and $13 \mathrm{~Hz}$ ("acrosshemifield left") or 11 and $14 \mathrm{~Hz}$ ("across-hemifield right"), respectively, during "across-hemifield" conditions. Note that the labels "left" and "right" refer to the hemifield location of the intermediate position between the attended LEDs. During "within-hemifield" conditions, they attended to pairs of LEDs that flickered at 10 and $14 \mathrm{~Hz}$ in the left visual hemifield ("within-hemifield left") or to pairs of LEDs flickering at 9 and $13 \mathrm{~Hz}$ in the right visual field ("within-hemifield right"), respectively (see Figure 1A). Participants were instructed to maintain gaze on the centrally presented fixation cross while they covertly shifted their attention to the cued pairs of LEDs and to avoid blinks during the stimulation periods.

\section{Procedure}

Each trial started with a brief flash (60 msec) of the two taskrelevant LEDs followed by a randomly chosen idle time of 150 or $250 \mathrm{msec}$. Subsequently, all LEDs flickered for a duration of 5200 msec. Participants were allowed to blink during a 1200-msec break following flicker stimulation.

The behavioral task entailed accurate and quick responses to simultaneously occurring brief interruptions in flickering of to-be-attended LED pairs (targets). Interruptions lasted 180 msec during which the task-relevant LED pair remained "on," that is, nonflickering. The short duration of target events was chosen to rule out that participants adopted a strategy to attend to one location and then rapidly switched attention to the other. Empirical evidence suggests that the minimum switching time under such conditions is in the range of 200-500 msec (Müller et al., 2003; Peterson \& Juola, 2000; Duncan, Ward, \& Shapiro, 1994; Weichselgartner \& Sperling, 1987; Reeves \& Sperling, 1986). Similar events that involved unattended LEDs had to be ignored (distractors). Distractor events could be composed of one attended LEDs and one unattended LED or two unattended LEDs, which allowed for a thorough control of whether participants deployed their attention to the cued LED pair throughout the stimulation period. Responses to distractor events were considered incorrect. In trials containing targets and distractors, one, two, or three of these events were presented at fixed pseudorandom onsets at 500, 1200, 2200, 3200 , or $4200 \mathrm{msec}$ after stimulus onset. In pilot experiments (10 participants), we adjusted the difficulty of the task by decreasing target luminance as to yield an average hit rate of about $80 \%$.

The experiment was run in 12 blocks with 40 trials each. Experimental conditions, namely, "within-hemifield left," "within-hemifield right," "across-hemifield left," and "across-hemifield right" splits of attention, alternated in a block-wise manner. Blocks were administered in pseudorandomized order. Half of all trials of the experiment contained no events; these were submitted to the analysis of SSVEP amplitudes. Of the remaining 50\% of trials, 75\% contained target events in attended LED pairs, and 25\% contained distractor events either in unattended LED pairs or in pairs that involved one attended and one unattended LEDs. Targets and distractor events were pseudorandomly assigned to attended and unattended LED pairs. Participants responded via button press. After half of the experimental blocks, participants changed the responding hand, and sequence of hand usage was balanced across individuals. Before the experimental session, participants performed a training session during which they familiarized themselves with the task in 12 training blocks of 40 trials each. In contrast to experimental blocks, each trial either contained a target or a distractor event to facilitate task learning.

\section{Data Acquisition}

Data were acquired in an electrically shielded chamber. EEG was recorded from $64 \mathrm{Ag}-\mathrm{AgCl}$ cap-mounted electrodes using an ActiveTwo amplifier system (BioSemi, Amsterdam, The Netherlands) set to a sampling rate of $256 \mathrm{~Hz}$. Four additional electrodes measured vertical and horizontal EOG to monitor eye movements and blinks.

\section{Data Analysis}

\section{Behavioral Data}

Responses that followed target event onsets within a time range of 200-800 msec were considered hits. Similar responses after distractor onsets were considered false alarms. RTs were only analyzed for hits. In addition, we evaluated the accuracy of given responses by means of hit rates.

We controlled for a left visual field advantage associated with a right-hemispheric dominance that is recurrently reported when healthy individuals perform visuospatial attention tasks (for a review, see Corbetta \& Shulman, 2011). Paired $t$ tests compared left versus right visual hemifields. Given that we found no significant differences between conditions "across-hemifield left" versus "acrosshemifield right" as well as between conditions "withinhemifield left" versus "within-hemifield right" (see Results), we collapsed across left and right to yield aggregate conditions "across-hemifield" and "within-hemifield." Again, paired $t$ test compared collapsed RTs and hits between "across-hemifield" and "within-hemifield" conditions. We abstained from further analyses of false alarm rates because participants produced only few false alarms on average (see Tables 1 and 2).

\section{EEG Data Preprocessing}

Preprocessing and analysis of collected data made use of the MATLAB-based EEGLAB toolbox (EEGLAB, UC San Diego; Delorme \& Makeig, 2004) in combination 
with custom-written routines. Epochs of 5000-msec duration, starting with stimulus onset, were extracted from continuous EEG recordings. Only target- and distractorfree trials were included in data analysis because rhythmic stimulation was interrupted during static event presentation. Epochs containing eye movements that exceeded a threshold of $25 \mu \mathrm{V}\left(\sim 2^{\circ}\right.$ of visual angle) were excluded from SSVEP analysis. Remaining epochs were submitted to an extended version of the statistical control of artifacts in dense array EEG/MEG (SCAD) studies (Junghöfer, Elbert, Tucker, \& Rockstroh, 2000). SCAD studies served to identify artifacts based on statistical parameters of the data and to correct artifact-contaminated channels by means of spherical spline-based interpolation. Epochs exceeding a maximum of 12 artifact-contaminated channels were rejected. Average rejection rate across conditions and participants was $7 \%(S E M=1.74 \%)$. The number of remaining epochs for each participant did not vary systematically between the four conditions (main effect, condition: $F(3,13)=0.63, p=.60$ ). Artifact-free epochs were rereferenced to average reference and averaged for each condition and participant, separately.

\section{EEG Data Analysis}

Fourier transforms of averaged artifact-free epochs within 500-4500 msec after flicker onset estimated SSVEP amplitudes at respective driving frequencies. The first $500 \mathrm{msec}$ of stimulation were excluded from analysis to avoid by ERPs to flicker onset and SSVEP build up. Grand-averaged isocontour voltage maps of SSVEP amplitudes averaged across conditions for each frequency were visually inspected to select electrode clusters covering local peaks. As expected, SSVEP amplitude maxima were found at parietal-occipital sites (see Figure 2A). Each frequencyspecific maximum lateralized to the hemisphere contralateral to its driving stimulus. To account for these retinotopic scalp projections of cortical SSVEP generators, we selected a cluster consisting of seven electrodes covering left occipito-parietal areas (O1, I1, PO3, PO7, P5, P7, and P9) to analyze SSVEPs driven by right-hemifield stimuli (9, 11, and $13 \mathrm{~Hz}$ ). Another cluster covering a similar area over the right cerebral hemisphere (O2, I2, PO4, PO8, P6, P8, and P10) served to analyze SSVEPs driven by left-hemifield stimuli $(10,12$, and $14 \mathrm{~Hz}$; see Figure 2A). Note that these electrode clusters coincided with topographic SSVEP amplitude peaks only approximately for some frequencies because we opted for a more conservative left-right symmetrical arrangement that accentuated the specific contribution of the respective hemisphere contralateral to the stimulated hemifield for each of the six stimuli. Amplitudes averaged across respective electrodes, for each frequency separately, entered statistical analysis.

A grand-averaged spectrum (averaged across electrodes O1 and O2) showed distinctive peaks at all six LED flicker frequencies that were present in all four experimental

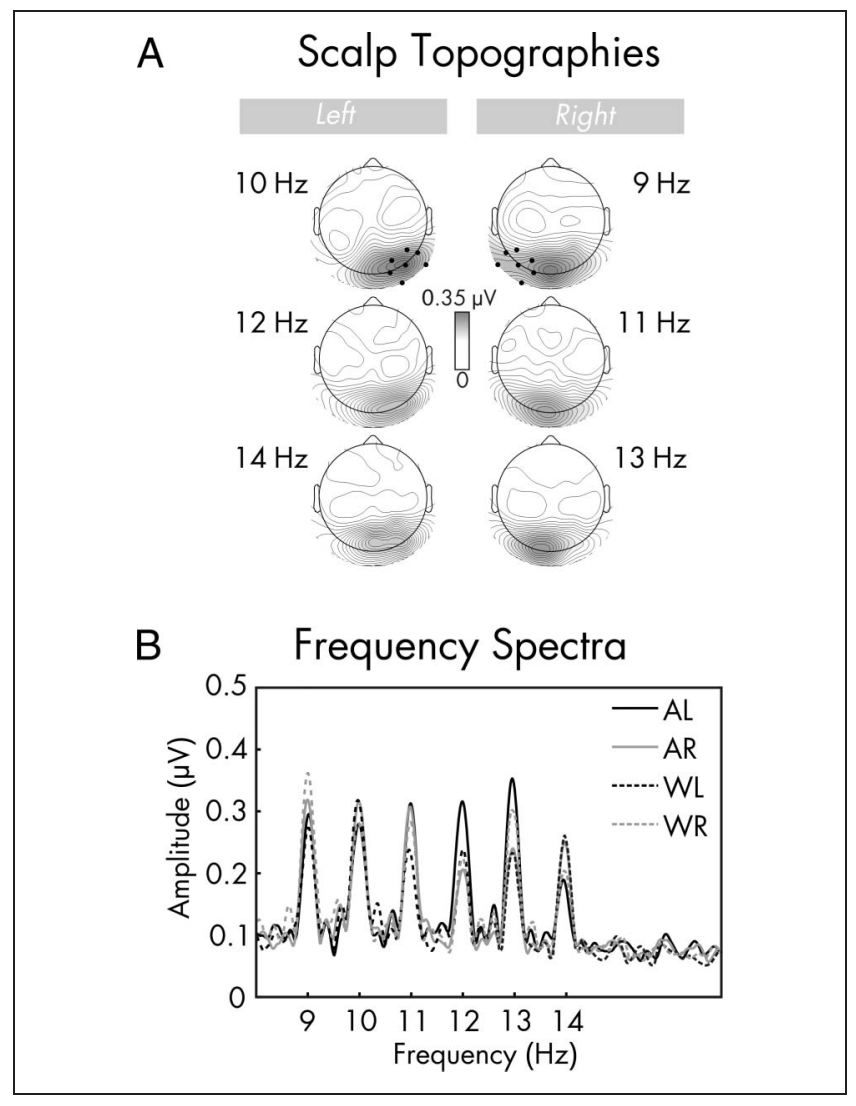

Figure 2. (A) Grand-averaged isocontour voltage maps for all frequencies. Voltage maps are derived from data in the time window of 500-4500 msec after stimulus onset. Left column contains maps for $10 \mathrm{~Hz}, 12 \mathrm{~Hz}$, and $14 \mathrm{~Hz}$ that were presented in the left visual field, right column depicts toporaghies for frequencies $9 \mathrm{~Hz}, 11 \mathrm{~Hz}$, and $13 \mathrm{~Hz}$ that were used during stimulation in the right hemifield. Black dots at posterior electrode sites highlight the electrodes chosen for statistical analysis. (B) Grand-averaged amplitude spectrum in the time window of 500-4500 msec after stimulus onset. Here, data averaged across electrodes $\mathrm{O} 1$ and $\mathrm{O} 2$ are plotted exemplarily for "across-hemifield left" (AL), "across-hemifield right" (AR), "within-hemifield left" (WL), and "within-hemifield right" (WR) conditions.

conditions (see Figure 2B). Further analyses were based on respective peak SSVEP amplitudes.

\section{Gain Effects on SSVEP Amplitude}

Common practice recommends a normalization of individual absolute SSVEP amplitudes (Keitel et al., 2013; Störmer et al., 2013; Andersen, Fuchs, \& Müller, 2011). This normalization serves two main purposes: It strips amplitudes of their large interindividual variance while retaining the net effect of SSVEP modulation by some experimental factor (here, spatial attention). Furthermore, normalization allows unbiased comparisons of effects between frequencies, and ultimately, normalized amplitudes can be pooled across different stimulation frequencies. To this end, amplitudes of each condition are frequently divided by a common denominator that 
can be the individual mean across conditions. In our case, however, normalizing by the mean was not feasible because different stimulus positions were subject to different patterns of attentional allocation across conditions (see Figure 3). For example, the leftmost LED, flickering at $9 \mathrm{~Hz}$ (=Position 3), was attended in the "within-hemifield left" condition only, whereas the 13-Hz LED, only left of the vertical meridian (=Position 1), was attended in "withinhemifield left" and "across-hemifield right" conditions. Given that attention increases SSVEP amplitudes, the mean across conditions for each respective stimulus position would thus have been a biased rather than a common norm.

Instead, we opted for a different approach: We divided individual SSVEP amplitudes from each condition by respective amplitudes from conditions in which participants were attending to the opposite hemifield (compare Figure 3B). More specifically, SSVEP amplitudes corresponding to stimuli in the left visual field $(9,11$, and $13 \mathrm{~Hz}$ ) in "within-hemifield left," "across-hemifield left," and "across-hemifield right" conditions were divided by amplitudes from "within-hemifield right" condition for each frequency separately (Figure 3B). Conversely, SSVEP amplitudes corresponding to stimuli in the right visual field $(14,12$, and $10 \mathrm{~Hz})$ in "within-hemifield right," "across-hemifield left," and "across-hemifield right" conditions were divided by amplitudes from "within-hemifield left" condition. SSVEP amplitudes of respective "withinhemifield" conditions served as common denominator for each respective frequency because the positions of the driving LEDs in contralateral hemifields were completely ignored. Note that any form of normalization requires the stimulation to drive SSVEPs of considerable amplitude, which can be safely assumed in our case as exemplified in Figure 2B.

Further justifying our approach, an earlier study found that SSVEPs, driven by stimuli in the unattended hemifield, were excluded from attentional modulation as well as active suppression (Müller, Teder-Sälejärvi, et al., 1998).
Finally, normalized amplitudes allowed us to directly compare SSVEP amplitude modulation, that is, attentional gain effects, between stimulus positions and conditions.

It should be noted that this normalization procedure excluded the data of the condition used as denominator from our analyses because dividing an amplitude value by itself yields 1 . This approach thus precluded direct analyses of the processing of (ignored) left-hemifield stimuli in the "within-hemifield right" condition and vice versa. Yet, crucially, it still allowed testing our hypotheses that focused on gain effects at attended and intermediate stimulus positions.

\section{Gain Effects: Statistical Analyses}

Our normalization procedure naturally yielded amplitude values with a left-skewed distribution and a mean of 1 . By taking the logarithm of normalized amplitudes, multiplied by 20 , we converted normalized amplitudes to decibel scale and, thus, approximated a normal distribution (skew minimized) that better met the requirements of parametric statistical procedures.

In a first step, we entered individual log-normalized values for each of the six LED locations into a threeway repeated-measures ANOVA comprising the factors of Hemifield (left, right), Condition (Positions 1 and 3 attended, Position 2 attended, Position 1 attended; see Figure $3 \mathrm{~A}$ and $\mathrm{B})$, and Position ( $1=$ closest to the vertical meridian, 2 and 3 = farthest from the vertical meridian; see Figure 3A). Note that, for the latter factors, data of positions in the right hemifield were mirrored to obey the same order as the left-hemifield positions. This ANOVA revealed that attentional modulation was independent of the factor Hemifield (see Results). Thus, in further analyses, data were collapsed across left- and right-hemifield stimuli.

From hemifield-collapsed data, we derived "pure" profiles of the spatial distribution of attentional gain effects
Figure 3. (A) Taxonomy of stimulus position exemplified for one hemifield (positions are mirror symmetric in the other hemifield). Position ("Pos") 1 is located closest to and Position 3 is located furthest from the vertical hemifield boundary. (B) All four experimental conditions demand a different allocation of attentional resources within each hemifield: Positions 1 and 3 are attended when attention is split within

the depicted hemifield (as shown in the simplified stimulus arrangement adapted from A), Position 2 is attended when attention is split across hemifields, and Position 1 is the intermediate position. Position 1 is attended during splits across hemifields when the intermediate position falls into the other hemifield. When participants split their attention within the other hemifield, no position is attended (dashed gray box). In data analyses, SSVEP amplitudes of this condition were used as a common denominator in normalizing amplitudes of the other three conditions (see Methods for details). (C) Conditions in which Positions 1 and 3 are attended deliver spatial profiles of "within-hemifield" splits. Similar profiles of "across-hemifield" splits are reconstructed from the remaining two conditions (excluding the norm condition, see B) whereby the attended Position 1 (third condition) represents the attended location beyond the hemifield boundary (dashed black line). 
("spatial profiles") by considering only both attended and the intermediate position in "within-hemifield" (Positions 1 and 3 attended) and "across-hemifield" conditions. To this end, we merged data of "across-hemifield" condition amplitude profiles while only retaining Positions 2 and 1 (intermediate) from condition "Position 2 attended" and completing the profile with Position 1 of condition "Position 1 attended" (see Figure 3C).

These profiles afforded testing our hypotheses about the differences between "within-hemifield" and "acrosshemifield" splits. In a first step, profiles were submitted to a two-way ANOVA with factors of Split type (within vs. across) and Position (1, 2, and 3). Subsequent specific contrasts (paired two-tailed $t$ tests) between "withinhemifield" versus "across-hemifield" splits targeted (1) the average attentional gain at attended stimulus positions, (2) the modulation at intermediate (i.e., between the attended) stimulus positions, and finally, (3) the average difference in attentional modulation between attended and intermediate stimulus positions. Additional two-tailed $t$ tests against zero were conducted to evaluate whether specific modulations were substantial.

In both ANOVAs conducted, we corrected probabilities to control for violations of sphericity by adjusting the degrees of freedom (Greenhouse \& Geisser, 1959) and report original degrees of freedom, corrected $p$ values $\left(p_{\mathrm{GG}}\right)$, and the correction coefficient epsilon $\left(\varepsilon_{\mathrm{GG}}\right)$. Post hoc tests (paired two-tailed $t$ tests) were conducted where appropriate.

\section{RESULTS}

\section{Behavioral Data}

An overview of behavioral measures is given in Table 1. Behavioral performance in left versus right visual hemifields neither differed in response time ("across-hemifield left" vs. "across-hemifield right": $t(12)=-1.11, p=.28$; "within-hemifield left" vs. "within-hemifield right": $t(12)=$ $-0.60, p=.55$ ) nor in hit rates ("across-hemifield left" vs. "across-hemifield right": $t(12)=0.10, p=.91$; "withinhemifield left" vs. "within-hemifield right": $t(12)=-0.07$, $p=.94$ ). Participants were responding faster ("across hemifields" vs. "within hemifields": $t(12)=-3.85, p<$ $.005)$ and more accurately to target events on LED pairs that were positioned across hemifields (hit rate "across hemifields" vs. "within hemifields": $t(12)=3.05, p=.01)$.

\section{SSVEP Amplitudes: Gain Effects}

Figure 2B illustrates the grand-averaged spectrum at electrodes $\mathrm{O} 1$ and $\mathrm{O} 2$ for the four experimental conditions. Distinct amplitude peaks are visible at stimulation frequencies of $9,10,11,12,13$, and $14 \mathrm{~Hz}$, respectively. Absolute peak amplitudes of condition-specific grand averages are plotted in Figure 4A. From these amplitudes, we derived indices of attentional modulation, that is, gain effects, at each stimulus position and in each of three possible configurations of attentional allocation within one hemifield ("Positions 1 and 3 attended," "Position 2 attended," and "Position 1 attended"; see Figure 3B) by means of a normalization procedure.

A first three-way repeated-measures ANOVA of Gain effects (decibel-scaled normalized amplitudes) revealed that attentional modulation was comparable in both visual hemifields (main effect, Hemifield: $F(1,12)=0.33$, $p=.58$ ), across all positions (main effect, Position: $F(2,24)=$ $0.12, p=.89$ ), and between conditions (main effect, Condition: $F(2,24)=1.06, p=.36)$. However, attentional gain depended on a systematic interaction of factors condition and position $\left(F(4,24)=5.32, p_{\mathrm{GG}}<.005, \varepsilon_{\mathrm{GG}}=0.729, \eta^{2}=\right.$ 0.05 ). Further interactions were negligible (all $F \mathrm{~S}<1$ ).

These results gave two important precursors for subsequent analyses: (1) The lack of influence of the factor hemifield allowed for a collapsing of gain effects across left and right hemifields. Figure 4B shows collapsed gain effects for the three conditions "Positions 1 and 3 attended," "Position 2 attended," and "Position 1 attended." (2) The Condition $\times$ Position interaction justified a closer investigation of variations in gain effects across conditions. In fact, post hoc comparisons elucidated that this interaction stemmed from systematic simple interactions, that is, terms that expressed differences of attentional gain in adjacent stimulus positions between two given conditions: Specifically, the difference in attentional modulation

Table 1. Behavioral Data of the Main Experiment

\begin{tabular}{llrr}
\hline Split Condition & Mean RTS & Hit Rate & False Alarm Rate \\
\hline Across left & $502 \mathrm{msec}($ SEM $=12)$ & $86.56($ SEM $=2.76)$ & $2.02($ SEM $=0.52)$ \\
Across right & $511 \mathrm{msec}($ SEM $=10)$ & $86.09($ SEM $=3.87)$ & $1.77($ SEM $=0.43)$ \\
Within left & $520 \mathrm{msec}($ SEM $=12)$ & $77.38($ SEM $=5.61)$ & $1.40($ SEM $=0.10)$ \\
Within right & $525 \mathrm{msec}($ SEM $=10)$ & $77.73($ SEM $=3.89)$ & $3.73($ SEM $=0.68)$ \\
Across hemifields & $506 \mathrm{msec}($ SEM $=10)$ & $86.33($ SEM $=2.59)$ & $1.89($ SEM $=0.40)$ \\
Within hemifields & $523 \mathrm{msec}($ SEM $=11)$ & $77.55($ SEM $=4.16)$ & $2.57($ SEM $=0.35)$ \\
\hline
\end{tabular}

Displayed are mean RTs, hit rates, and false alarm rates, including $S E M$, for the left-right comparison (Rows 1-4) and the different-hemifield advantage (Rows 5 and 6). 


\section{A SSVEP Peak Amplitudes}
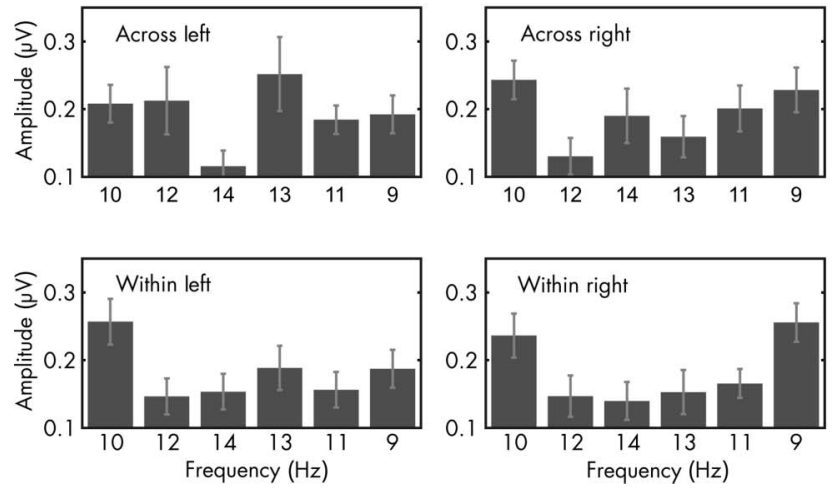

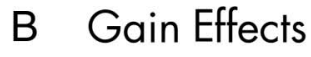

C Spatial Profiles

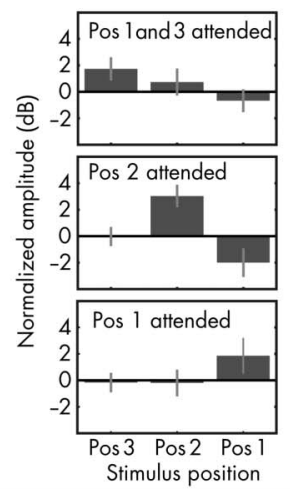

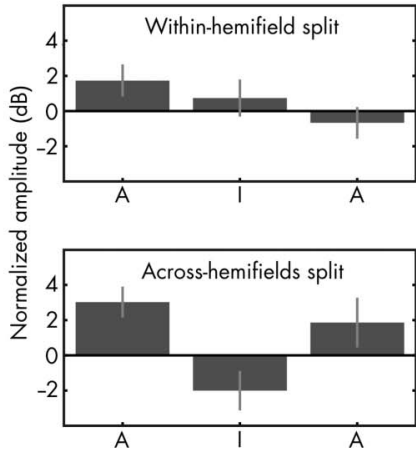

Figure 4. (A) Absolute amplitudes at all frequencies in the display for split conditions "across-hemifield left" (attend 12 and $13 \mathrm{~Hz}$ ), "across-hemifield right" (attend 11 and $14 \mathrm{~Hz}$ ), "within-hemifield left" (attend 10 and $14 \mathrm{~Hz}$ ), and "within-hemifield right" (attend 9 and $13 \mathrm{~Hz}$ ). Error bars indicate SEM. (B) Normalized amplitudes (in dB) express gain effects at each stimulus position in three conditions derived as described in Figure 3B. Data is collapsed across left and right hemifield stimulation. Normalized amplitudes (arbitrary unit) giving rise to the activity profile at attended (A) and intermediate (I) positions, when participants split their spotlight of attention across (left barplot) and within (top barplot) hemifields. Error bars in A, $\mathrm{B}$, and $\mathrm{C}$ represent SEM.

between Positions 3 and 2 (see Figures 3 and 4B) varied systematically between conditions "Positions 1 and 3 attended" and "Position 2 attended" $(t(12)=-2.50, p<$ $.05)$. The same held true for gain effects of Positions 2 and 1 between conditions "Positions 1 and 3 attended" and "Position 2 attended" $(t(12)=2.31, p<.05)$ as well as between conditions "Position 2 attended" and "Position 1 attended" $(t(12)=-4.47, p<.001)$. Only the comparison of differences in Positions 3 and 2 between conditions "Position 2 attended" and "Position 1 attended" escaped significance $(t(12)=1.99, p=.07)$. This was likely because of the unique situation that Positions 3 and 2 were both unattended in the "Position 1 attended" condition (see Figures $3 \mathrm{~B}$ and $4 \mathrm{~B}$ ) and was irrelevant to further analyses.

\section{Spatial Profiles of Attentional Gain}

For a direct comparison of the spatial distribution of gain effects during attentional splits within versus across hemifields, we reconstructed so-called spatial profiles consisting of only the most relevant positions: attendedintermediate-attended (see Figure 3C and Methods). A two-way ANOVA conducted on these profiles, as depicted in Figure $4 \mathrm{C}$, corroborated the variation in gain effects between the three positions (main effect, Position: $F(2$, $\left.24)=4.41, p_{\mathrm{GG}}<.05, \varepsilon_{\mathrm{GG}}=0.974, \eta^{2}=0.09\right)$ but did not substantiate an overall difference between "withinhemifield" and "across-hemifield" splits of attention (main effect, Split type: $F(1,12)=0.16, p=.69$ ). However, for each position, gain effects varied differentially between conditions as shown by a significant interaction of factors Position and Split type $\left(F(2,24)=5.66, p_{\mathrm{GG}}<.05, \varepsilon_{\mathrm{GG}}=\right.$ $0.495, \eta^{2}=0.08$ ).

At first glance, the lack of a main effect of Split type seemed to contradict our hypotheses, but the ANOVA, as carried out here, was likely insensitive to such an effect because of the more pronounced yet opposed gain effects on attended and intermediate positions in "acrosshemifield" splits as compared with "within-hemifield" splits (see Figure 4C). Instead, differences between both split types could be substantiated by specific contrasts.

As predicted by the notion of hemisphere-specific independent resources, average gain effects on attended stimulus positions were greater in "across-hemifield" than "within-hemifield" splits $(t(12)=-2.42, p<.05)$. Moreover, only during "across-hemifield" splits was the average gain substantial, that is, greater than zero $(t(12)=2.40$, $p<.05)$. We did not find a systematic enhancement of attended stimulus processing during "within-hemifield" splits $(t(12)=0.83, p=.42)$.

Assuming hemisphere-specific limited resources also predicted greater competition for processing when attention was split within one hemifield, we hypothesized that this may lead to impeded filtering of possibly distracting stimuli at intermediate positions. Although the difference in attentional modulation at intermediate positions ("within hemifields" minus "across hemifields") did not reach statistical significance $(t(12)=1.73, p=.11)$, we found that, when taking into account the average distance of gain effects between attended and intermediate positions (attended minus intermediate), again, "across-hemifield" splits generated a more pronounced profile of attentional modulation than "within-hemifield" splits $(t(12)=-3.19$, $p<.01)$. Supporting this finding, only the distance during "across-hemifield" splits was greater than zero ("across hemifields": $t(12)=4.74, p<.001$; "within hemifields": $t(12)=-0.15, p=.89)$.

\section{Mexican Hat Simulation}

Encouraged by our findings, we tested whether the spatial gain profiles match with the assumptions of the 
competitive content maps account (Franconeri et al., 2013). To this end, we simulated the distribution of attentional resources among stimuli numerically for "across-hemifield" and "within-hemifield" conditions. Note that Franconeri et al. simplified the profile of the attentional spotlight to a discrete facilitatory center region surrounded by a suppressive region (Figure 5A). Studies into the fine-grained spatial structure of the spotlight, however, suggest a gradual Mexican hat-shaped profile (Boehler, Tsotsos, Schoenfeld, Heinze, \& Hopf, 2011; Müller, Mollenhauer, Rösler, \& Kleinschmidt, 2005). For our simulation, we assumed the following: Dependent on their spatial extent, two Mexican hat spotlights form a weighted sum when attention focuses on two close-by stimuli (Bahcall \& Kowler, 1999). The hemifield boundary impedes (in our case, interrupts) this summation process. Previous studies pinpoint the trough corresponding to the maximum surround suppression at an eccentricity of between $1.4^{\circ}$ (Boehler et al., 2011) and $2.5^{\circ}$ of visual angle (Müller et al., 2005) from the facilitatory center. As a first step, we thus derived the potential shape of a unitary spotlight from the spatial profile of attention effects in the "across-hemifield" condition (Figure 4B). Here, the peak gain effect amounted to $\sim 50 \%$ (3 dB) for both attended stimuli. The intermediate stimulus neither experienced facilitation nor suppression ( $t$ test against zero: $t(12)=-1.79, p=.1$ ), suggesting that it fell close to the zero crossing between center and surround (Figure 5B). Given the spatial separation of $2^{\circ}$ of visual angle between stimulus positions, we were thus able to estimate the width and the height of the Mexican hat spotlight. To model the overlap of two spotlights in the "within-hemifield" condition, their spatial profiles were summed and divided by a factor of 2

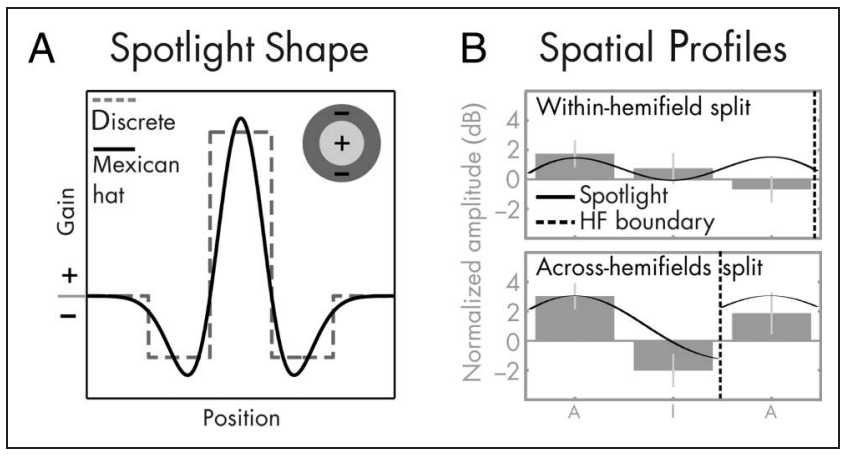

Figure 5. Mexican hat fit of spatial gain profiles. (A) Schematic attentional spotlight with facilitatory center $(+)$ and suppressive surround (-). Inset shows discrete profile as used by Franconeri et al. (2013). Gray dashed line $=1$-D spatial profile with discrete centersurround. Black solid line = gradual Mexican hat profile. (B) Overlay of Mexican hat simulation results on Figure 4B (gray bars). Black solid lines depict simulated spotlights that are separated by the hemifield (HF) boundary (vertical dashed line) in "across" conditions (left) and merged in "within" conditions (right). Note that the gain profile does not extend into suppressive regions (below horizontal gray line) at the intermediate position for "within" conditions. to account for the division of hemisphere-specific resources between locations.

Results, illustrated in Figure 5B (right), closely resemble the profile obtained from SSVEP amplitude modulation in "within-hemifield" conditions: Attenuation of gain effects at attended positions relative to "across-hemifield" conditions fits our data. Moreover, merging spotlights produce a small nonsuppressive region between their peaks that is in line with a diminished filtering of task-irrelevant intermediate distractors during "within-hemifield" splits.

\section{Behavioral Control Experiment}

In our study, we used a wide range of tagging frequencies (9-15 Hz). Therefore, it was necessary to rule out the possibility that different tagging frequencies led to differences in perceived stimulus salience and, as a consequence, to a stimulus-driven bias in attentional selection. To this end, we ran a behavioral control experiment in which all six LEDs were flickered at the same frequency. If flicker frequency would have an influence on the selection process or, in other words, participants would have attended to the feature flicker frequency rather than to location, we would have expected a different pattern of behavioral results.

Eight individuals who participated in the EEG experiment volunteered for the control study. The experimental design and procedure were identical to the main experiment with the exception that LEDs of all six locations flickered at $12 \mathrm{~Hz}$. Twelve blocks of 40 trials were run. Each trial contained either target (75\% of trials) or distractor (25\% of trials) events. Behavioral data were acquired and analyzed as described for the main experiment. In addition, we compared the differences in "acrosshemifield" and "within-hemifield" performances, that is, the different-hemifield advantage, between the main and control experiments by means of two-tailed paired $t$ tests. Contrasts were performed on data of eight participants who took part in both experiments.

\section{Results: Control Experiment}

Behavioral data of the control experiment are summarized in Table 2. We replicated the pattern of results of the main experiment with no differences in performance between left compared with right hemifields for either RTs ("across-hemifield left" vs. "across-hemifield right": $t(7)=1.00, p=.34$; "within-hemifield left" vs. "withinhemifield right": $t(7)=0.07, p=.94)$ or hit rate ("acrosshemifield left" vs. "across-hemifield right": $t(7)=1.56$, $p=.16$; "within-hemifield left" vs. "within-hemifield right": $t(7)=1.81, p=.11$ ). Again, RTs during average performance across hemifields were shorter ("across hemifields" vs. "within hemifields": $t(7)=-6.10, p<.001)$, and hit rates were higher ("across hemifields" vs. "within hemifields": $t(7)=3.22, p=.01)$. Comparisons of RTs and hit rates between experiments were insignificant (RTs: $t(7)=-0.32$, 
Table 2. Mean RTs, Hit Rates, and False Alarm Rates with SEM during Performance in the Behavioral Control Experiment

\begin{tabular}{llrr}
\hline Split Condition & Mean RTs & Hit Rate & False Alarm Rate \\
\hline Across left & $500 \mathrm{msec}($ SEM $=14)$ & $89.78($ SEM $=1.65)$ & $2.47($ SEM $=0.62)$ \\
Across right & $494 \mathrm{msec}($ SEM $=16)$ & $82.55($ SEM $=4.50)$ & $2.16($ SEM $=0.51)$ \\
Within left & $525 \mathrm{msec}($ SEM $=14)$ & $80.57($ SEM $=3.94)$ & $1.91($ SEM $=0.37)$ \\
Within right & $524 \mathrm{msec}($ SEM $=18)$ & $74.12($ SEM $=4.20)$ & $4.29($ SEM $=0.65)$ \\
Across hemifields & $497 \mathrm{msec}($ SEM $=15)$ & $86.17($ SEM $=1.09)$ & $1.57($ SEM $=0.07)$ \\
Within hemifields & $524 \mathrm{msec}($ SEM $=16)$ & $77.35($ SEM $=2.01)$ & $1.26($ SEM $=0.12)$ \\
\hline
\end{tabular}

First four rows report the data for the performance in the left hemifield compared with the right hemifield. Last two rows show the data for the investigation of the different-hemifield advantage.

$p=.76$; hit rates: $t(7)=0.52, p=.62$ ). Collectively, these results rule out that the use of different flicker frequencies influenced the results of the main experiment regarding the different-hemifield advantage.

\section{DISCUSSION}

We investigated differences in the ability to divide the spotlight of attention between noncontiguous locations within or across left and right visual hemifields. Our paradigm, employing the frequency tagging method, allowed us to examine the simultaneous allocation of attention to multiple distinct locations and thus the distribution of processing capacities across space in early visual cortex: We manipulated the deployment of attention in a multielement display comprising six LEDs that were positioned in left and right visual hemifields below the horizontal meridian. LEDs delivered continuous flicker stimulation for several seconds, which elicited oscillatory brain responses, SSVEPs, that provided an objective electrophysiological measure of individual visual stimulus processing.

\section{Distinct Gain Effects Support the Different-hemifield Advantage}

We derived specific hypotheses from the idea of independent resource pools per cerebral hemisphere (Sereno \& Kosslyn, 1991) and the competitive content maps account (Franconeri et al., 2013). In accord with hypotheses as well as with earlier studies on the different-hemifield advantage using fMRI (Kraft et al., 2011) and SSVEPs (Störmer et al., 2014; Walter et al., 2014), we found a pronounced attention effect for the two to-be-attended positions versus the intermediate to-be-ignored positions only when participants divided their attention across the left and right visual hemifields. There was no evidence for this gain effect in "within-hemifield" conditions. Behavioral data further confirmed a different-hemifield advantage with better task performance during "across-hemifield" compared with "within-hemifield" trials (Alvarez et al.,
2012; Kraft et al., 2005, 2007, 2011; Alvarez \& Cavanagh, 2005; Sereno \& Kosslyn, 1991).

Our results were unlikely influenced by a left visual field advantage that is associated with a right-hemispheric dominance (Corbetta \& Shulman, 2011) because early visual processing and behavioral performance were comparable in left and right hemifields. We can further exclude any bias introduced by tagging each stimulus with a different frequency because, in our control experiment, we used the same frequency for all stimuli and we replicated the patterns of behavioral performance obtained in the EEG experiment; again, participants' performance across hemifields was superior to performance within hemifields.

Furthermore, as previous experiments have acknowledged (McMains \& Somers, 2004; Müller et al., 2003), participants could not have performed the task by switching between both attended locations because targets were presented for $180 \mathrm{msec}$. Such a brief interval disallowed switching and processing information from two locations sequentially (Müller et al., 2003; Peterson \& Juola, 2000; Duncan et al., 1994; Weichselgartner \& Sperling, 1987; Reeves \& Sperling, 1986).

One might still argue that our results regarding neural gain are at least partially influenced by a difference in task difficulty. More specifically, "within-hemifield" splits reduced behavioral performance relative to "across-hemifield" splits, suggesting that former conditions required increased effort. However, a recent study, employing frequency tagging, showed that increased perceptual load-which in turn increases the effort necessary to deal with a task-does not affect and modulate SSVEPs (Hindi Attar \& Müller, 2012). Moreover, although thoroughly matching task difficulties between "across-hemifield" and "within-hemifield" conditions, Störmer et al. (2014) reported a difference in gain effects that nevertheless closely resembled ours. Finally, only event-free trials entered SSVEP analyses, which likely separated task-related from stimulus-related neural processes to some extent. Therefore, we are confident that the influence of task difficulty on differences in gain effects between "within-hemifield" and "across-hemifield" splits was negligible in our study. 


\section{Different-hemifield Advantage during Split Attention Relies on Distinct Spatial Gain Profiles}

The observed different-hemifield advantage during split attention likely results from differences in early visual processing between "across-hemifield" and "within-hemifield" conditions and endorses the notion of hemisphere-specific independent resource pools (Sereno \& Kosslyn, 1991): Each hemisphere attended to only one location during "across-hemifield" splits and was thus able to deploy all its resources toward processing of one specific stimulus. The same amount of resources had to be divided during "within-hemifield" splits explaining the accompanying diminished gain effects. Our observation of a substantial difference in gain effects between attended and intermediate positions during "across-hemifield" splits only, however, does not immediately follow from the assumption of independent resources and might be better captured by the competitive content maps account (Franconeri et al., 2013).

This account expresses the different-hemifield advantage (and thus the notion of hemisphere-specific resources) as a consequence of the discontinuity of suppressive stimulus interactions across the hemifield boundary. Put differently, suppressive surrounds of stimulus representation do not overlap during performance across hemifields and thus reduce mutual influences during attentional selection of relevant over irrelevant visual information (Franconeri et al., 2013). Less or no distractor exclusion is anticipated during within-hemifield performance; in this situation, all three stimuli fall within one hemispheric map and are in close spatial proximity. Therefore, suppressive surrounds of attended items can overlap leading to a weakening of target representations in visual cortex, as observed in the current results.

This claim is well in line with earlier findings of imaging studies that have reported a zone of inhibition surrounding the attentional focus (Hopf, Boehler, Schoenfeld, Heinze, \& Tsotsos, 2010; Boehler, Tsotsos, Schoenfeld, Heinze, \& Hopf, 2009; Hopf et al., 2006; Schwartz et al., 2005; Müller \& Kleinschmidt, 2004). Importantly, it has been reported that, in V4, attention can modulate center-surround interactions; attention to a given stimulus in the center of the receptive field of a neuron may weaken the suppression induced by a distractor in the surround (Sundberg, Mitchell, \& Reynolds, 2009). In turn, suppressive effects of a distractor on the center stimulus are increased as soon as attention is deployed toward the distractor in the surround.

In the current study, we further explored this centersurround inhibition by substituting the simplified discrete attentional spotlights of the competitive content maps account (Franconeri et al., 2013) with a more plausible gradual Mexican hat-shaped profile (Boehler et al., 2011; Müller et al., 2005; see Figure 5A) and simulated the distribution of attentional capacity among stimuli for multifocal "across-hemifield" and "within-hemifield" conditions.
Figure 5B summarizes the results indicating that our numerical simulation resembled the spatial profiles of SSVEP amplitude attentional modulation. As can be seen, a bifocal spotlight counteracts the contrast in gain effects between attended and intermediate positions in "withinhemifield" conditions. The present findings thus directly relate the different-hemifield advantage in splitting the spotlight of attention between noncontiguous locations to within-hemifield interactions of close-by Mexican hatshaped gain profiles (Boehler et al., 2011; Müller et al., 2005) and their disruption by the hemifield boundary (Franconeri et al., 2013; Sereno \& Kosslyn, 1991).

\section{Attentional Coamplification and Stimulus Integration}

Experimental findings similar to ours are sometimes discussed under the label of "stimulus integration." For instance, Large, Culham, Kuchinad, Aldcroft, and Vilis (2008) reported greater stimulus integration in "within" compared with "across" visual hemifield arrangements. In an fMRI study, they identified the lateral occipital region that seemingly integrates visual field information within a hemifield but not across hemifields. According to their view, the observed differences between "within-hemifield" and "across-hemifield" stimulus arrangements are a consequence of greater stimulus integration.

Kraft et al. (2011) came to a similar conclusion. In an fMRI study, they analyzed the BOLD responses in human visual cortex (V1-V4v) when participants were asked to perform a discrimination task at two locations that were separated by an intermediate to-be-ignored position. Hence, as in this study, observers had to split their spotlight of attention either across or within hemifields. The BOLD signal to the intermediate ignored position increased when the task had to be performed within hemifields as compared with across hemifields, thus revealing higher processing of intermediate ignored positions within than across hemifields. The authors concluded that their results provide evidence for greater stimulus integration within compared with across visual hemifields. Taken together, these findings point toward a close connection between the impeded capability to split the spotlight of attention between close-by stimuli within visual hemifields and their facilitated integration into visual objects.

\section{Within-hemifield Splits: Impossible or Just More Difficult?}

Malinowski et al. (2007) and Müller et al. (2003) investigated the allocation of attention to noncontiguous stimulus positions in two separate SSVEP studies: In the first experiment, individuals were asked to perform a splitattention task across hemifields (Müller et al., 2003); in the second experiment, the same stimuli were aligned within one hemifield (Malinowski et al., 2007). During "across-hemifield" performance in the first experiment, 
intermediate positions were filtered out successfully. Within-hemifield performance in the second experiment revealed the successful exclusion of intermediate locations during processing of relevant attended positions in the upper visual field but not in the lower visual field, further accompanied by a drop in performance in both conditions as compared with "across-hemifield" performance. The here reported finding of impaired visuospatial filtering within hemifields is in line with findings by Malinowski et al. (2007) because this experiment examined only lower visual field performance.

Nevertheless, McMains \& Somers (2004) reported weaker but significant exclusion of intermediate to-beignored positions within a cortical hemisphere, indicated by BOLD response modulation; they suggested that it is at least easier to split attention across hemifields. Although the present results lack this effect, we emphasize that they do not explicitly rule out splits of attention within visual hemifields. The discrepancy between this study and the studies of McMains \& Somers (2004) as well as Malinowski et al. (2007) might be because of two factors: (1) proximity of stimuli and (2) number of attended locations within visual quadrants. Both earlier experiments featured stimulation with two stimuli per quadrant (one target and one intermediate distractor).

In contrast, in the present work, three stimuli were densely aligned within each lower quadrant and might have led to increased stimulus interactions in early visual processing. This conclusion is supported by the finding that, during multiple-object tracking, performance is largely dependent on the spacing of objects (Franconeri, Jonathan, \& Scimeca, 2010). Systematically manipulating stimulus proximity and number of attended stimuli across and within hemifields may provide further insights in future studies.

Another possible reason for the divergence between McMains \& Somers (2004) and our findings might be that fMRI and SSVEPs index different aspects of attentional modulation in general. Previous studies measuring fMRI during spatial attention tasks (e.g., Pestilli, Carrasco, Heeger, \& Gardner, 2011; Murray, 2008; Buracas \& Boynton, 2007; Kastner, Pinsk, De Weerd, Desimone, \& Ungerleider, 1999) show substantially increased BOLD responses even when no stimulus is presented. A similar effect is absent in EEG-based measures of neural activity, such as SSVEPs and early stimulus-evoked responses (e.g., Itthipuripat, Ester, Deering, \& Serences, 2014; Itthipuripat, Garcia, Rungratsameetaweemana, Sprague, \& Serences, 2014; Kim, Grabowecky, Paller, Muthu, \& Suzuki, 2007; Di Russo, Spinelli, \& Morrone, 2001).

Finally, splitting attention within one hemifield could entail a relatively broad and uniform gain field (attention field, $\mathrm{AF}$ ) as proposed in the context of the Normalization Model of Attention (cf. Reynolds \& Heeger, 2009). Recently, it has been demonstrated that the size of this AF modulates behavioral performance (Herrmann, MontaserKouhsari, Carrasco, \& Heeger, 2010) and neural processing (measured using SSVEPs by Itthipuripat, Garcia, et al., 2014). For broad AFs and in situations with high-contrast, salient stimulation as in our experiment, the Normalization Model predicts only small to negligible gain effects. In contrast, splitting attention across hemifields could produce two distinct, well-circumscribed AFs. In that case and in line with our results, especially high-contrast stimuli experience considerable gain. Currently, however, it is unclear how the concept of attention as a normalization process models the hemifield boundary.

\section{Within-hemifield Splits: A Transient Phenomenon?}

Only recently, Itthipuripat et al. (2013) used frequency tagging to investigate temporal dynamics in split attentional spotlight tasks when all three stimuli were presented in left and right quadrants of the lower visual hemifield. When they analyzed the full stimulation period of $3200 \mathrm{msec}$, they found no differences in the processing of relevant compared with to-be-ignored intermediate distractor stimuli. This is well in line with the current results. However, they reported a short living exclusion of intermediate positions in a small time window (350-150 msec) before target stimuli that were correctly detected. The researchers concluded that, although the spotlight of attention can be divided across noncontiguous regions, this occurs only temporarily, as indicated by the brief period of target response enhancement. In our experiment, only trials without events and responses were used to investigate amplitude differences because, in contrast to the design of event trials in Itthipuripat et al. (2013), here, events (i.e., a "nonflickering" sequence of the LEDs interrupting the flicker sequence) disrupted the SSVEP. In consequence, an analysis of the period around the events could not be performed.

Various other factors may have contributed to the seemingly opposed results. Itthipuripat et al. (2013) asked participants to discriminate discrete gray rectangle-shaped marks that occurred simultaneously on stimuli at splitattended locations and within an area that involved less than $1 \%$ of stimuli. Critically, the white square distractor at the intermediate position never showed any marks, making it unnecessary for participants to inhibit conflicting information from that location. We thus assume that the intermediate stimulus influenced processing at attended locations to a lower extent as in this study, where (1) target and intermediate LEDs, as well as behaviorally relevant events, did not differ perceptually; (2) target events could occur at attended as well as to-be-ignored intermediate positions; and (3) stimuli were more densely spaced as they were relevant to the task in their entirety.

\section{Conclusion}

In summary we found that during divided spatial attention only splits across visual hemifields led to a successful exclusion of intermediate distractor positions. In 
turn, filtering relevant over irrevelant visual information was impeded when participants split their focus of attention within visual hemifields. These findings highlight the importance of hemisphere-specific early visual processing capacities in the ability to split the spotlight of attention across vs within hemifields and provide a direct a direct link to the different-hemifield advantage (Sereno and Kosslyn, 1991). Moreover, a simulation of the processing gain of densely spaced visual stimuli - using a Mexican hat conceptualization of the attentional spotlight - captured experimentally obtained differences in spatial gain profiles between across- and within-hemifield splits. This allowed us to connect recent findings on spotlight morphology with the ideas of competitive content maps (Franconeri et al., 2013) while assuming a fundamental role for the vertical meridian separating left and right visual hemifields.

\section{Acknowledgments}

We thank Renate Zahn, Anna Pfau, and Maike Salazar for their invaluable help during data acquisition and Ulrich Krebs (FriedrichWilhelm-Bessel-Institute, Bremen, Germany) for providing technical equipment and support for the LED stimulation apparatus. This experiment was realized using Cogent Graphics, developed by John Romaya at the Laboratory of Neuroscience at the Wellcome Department of Imaging Neuroscience. The research leading to these results has received funding from the Deutsche Forschungsgemeinschaft under grant MU972/20-1.

Reprint requests should be sent to Matthias M. Müller, Institute of Psychology, Leipzig University, Neumarkt 9-19, 04109 Leipzig, Germany, or via e-mail: m.mueller@rz.uni-leipzig.de.

\section{REFERENCES}

Alvarez, G. A., \& Cavanagh, P. (2005). Independent resources for attentional tracking in the left and right visual hemifields. Psychological Science, 16, 637-643.

Alvarez, G. A., Gill, J., \& Cavanagh, P. (2012). Anatomical constraints on attention: Hemifield independence is a signature of multifocal spatial selection. Journal of Vision, 12, $1-20$

Andersen, S. K., Fuchs, S., \& Müller, M. M. (2011). Effects of feature-selective and spatial attention at different stages of visual processing. Journal of Cognitive Neuroscience, 23, 238-246.

Andersen, S. K., Müller, M. M., \& Hillyard, S. A. (2011). Tracking the allocation of attention in visual scenes with steady-state evoked potentials. In M. I. Posner (Ed.), Cognitive neuroscience of attention (2nd ed., pp. 197-216). New York: Guilford.

Atmaca, S., Stadler, W., Keitel, A., Ott, D. V., Lepsien, J., \& Prinz, W. (2013). Prediction processes during multiple object tracking (MOT): Involvement of dorsal and ventral premotor cortices. Brain \& Behavior, 3, 683-700.

Awh, E., \& Pashler, H. (2000). Evidence for split attentional foci. Journal of Experimental Psychology: Human Perception and Performance, 26, 834-846.

Bahcall, D. O., \& Kowler, E. (1999). Attentional interference at small spatial separations. Vision Research, 39, 71-86.

Boehler, C. N., Tsotsos, J. K., Schoenfeld, M. A., Heinze, H.-J., \& Hopf, J.-M. (2009). The center-surround profile of the focus of attention arises from recurrent processing in visual cortex. Cerebral Cortex, 19, 982-991.
Boehler, C. N., Tsotsos, J. K., Schoenfeld, M. A., Heinze, H.-J., \& Hopf, J.-M. (2011). Neural mechanisms of surround attenuation and distractor competition in visual search. Journal of Neuroscience, 31, 5213-5224.

Buracas, G. T., \& Boynton, G. M. (2007). The effect of spatial attention on contrast response functions in human visual cortex. Journal of Neuroscience, 27, 93-97.

Corbetta, M., \& Shulman, G. L. (2011). Spatial neglect and attention networks. Annual Review of Neuroscience, 34, 569-599.

Delorme, A., \& Makeig, S. (2004). EEGLAB: An open source toolbox for analysis of single-trial EEG dynamics including independent component analysis. Journal of Neuroscience Methods, 134, 9-21.

Di Russo, F., Pitzalis, S., Aprile, T., Spitoni, G., Patria, F., Stella, A., et al. (2007). Spatiotemporal analysis of the cortical sources of the steady-state visual evoked potential. Human Brain Mapping, 28, 323-334.

Di Russo, F., Spinelli, D., \& Morrone, M. C. (2001). Automatic gain control contrast mechanisms are modulated by attention in humans: Evidence from visual evoked potentials. Vision Research, 41, 2435-2447.

Duncan, J., Ward, R., \& Shapiro, K. (1994). Direct measurement of attentional dwell time in human vision. Nature, 369, 313-315.

Eriksen, C. W., \& St. James, J. D. (1986). Visual attention within and around the field of focal attention: A zoom lens model. Perception \& Psychophysics, 40, 225-240.

Franconeri, S. L., Alvarez, G. A., \& Cavanagh, P. (2013). Flexible cognitive resources: Competitive content maps for attention and memory. Trends in Cognitive Sciences, 17, 134-141.

Franconeri, S. L., Jonathan, S. V., \& Scimeca, J. M. (2010). Tracking multiple objects is limited only by object spacing, not by speed, time, or capacity. Psychological Science, 21, 920-925.

Fuchs, S., Andersen, S. K., Gruber, T., \& Müller, M. M. (2008). Attentional bias of competitive interactions in neuronal networks of early visual processing in the human brain. Neuroimage, 41, 1086-1101.

Greenhouse, S. W., \& Geisser, S. (1959). On methods in the analysis of profile data. Psychometrika, 24, 1959.

Harasawa, M., \& Shioiri, S. (2011). Asymmetrical brain activity induced by voluntary spatial attention depends on the visual hemifield: A functional near-infrared spectroscopy study. Brain and Cognition, 75, 292-298.

Herrmann, K., Montaser-Kouhsari, L., Carrasco, M., \& Heeger, D. J. (2010). When size matters: Attention affects performance by contrast or response gain. Nature Neuroscience, 13, 1554-1559.

Hindi Attar, C., \& Müller, M. M. (2012). Selective attention to task-irrelevant emotional distractors is unaffected by the perceptual load associated with a foreground task. PLoS One, 7, e37186.

Hopf, J.-M., Boehler, C. N., Luck, S. J., Tsotsos, J. K., Heinze, H.-J., \& Schoenfeld, M. A. (2006). Direct neurophysiological evidence for spatial suppression surrounding the focus. Proceedings of the National Academy of Sciences, U.S.A., 103, 1053-1058.

Hopf, J.-M., Boehler, C. N., Schoenfeld, M. A., Heinze, H.-J., \& Tsotsos, J. K. (2010). The spatial profile of the focus of attention in visual search: Insights from MEG recordings. Vision Research, 50, 1312-1320.

Itthipuripat, S., Ester, E. F., Deering, S., \& Serences, J. T. (2014). Sensory gain outperforms efficient readout mechanisms in predicting attention-related improvements in behavior. Journal of Neuroscience, 34, 13384-13398.

Itthipuripat, S., Garcia, J. O., Rungratsameetaweemana, N., Sprague, T. C., \& Serences, J. T. (2014). Changing the spatial 
scope of attention alters patterns of neural gain in human cortex. Journal of Neuroscience, 34, 112-123.

Itthipuripat, S., Garcia, J. O., \& Serences, J. T. (2013). Temporal dynamics of divided spatial attention. Journal of Neurophysiology, 109, 2364-2373.

Jans, B., Peters, J. C., \& De Weerd, P. (2010). Visual spatial attention to multiple locations at once: The jury is still out. Psychological Review, 117, 637-684.

Junghöfer, M., Elbert, T., Tucker, D. M., \& Rockstroh, B. (2000). Statistical control of artifacts in dense array EEG/MEG studies. Psychophysiology, 37, 523-532.

Kahneman, D. (1973). Attention and effort. The American Journal of Psychology (Vol. 88, p. 339). Englewood Cliffs, NJ: Prentice-Hall.

Kastner, S., Pinsk, M. A., De Weerd, P., Desimone, R., \& Ungerleider, L. G. (1999). Increased activity in human visual vortex during directed attention in the absence of visual stimulation. Neuron, 22, 751-761.

Kastner, S., \& Ungerleider, L. G. (2001). The neural basis of biased competition in human visual cortex. Neuropsychologia, 39, 1263-1276.

Keitel, C., Andersen, S. K., \& Müller, M. M. (2010). Competitive effects on steady-state visual evoked potentials with frequencies in- and outside the alpha band. Experimental Brain Research, 205, 489-495.

Keitel, C., Andersen, S. K., Quigley, C., \& Müller, M. M. (2013). Independent effects of attentional gain control and competitive interactions on visual stimulus processing. Cerebral Cortex, 23, 940-946.

Kim, Y. J., Grabowecky, M., Paller, K. A., Muthu, K., \& Suzuki, S. (2007). Attention induces synchronization-based response gain in steady-state visual evoked potentials. Nature Neuroscience, 10, 117-125.

Kraft, A., Kehrer, S., Hagendorf, H., \& Brandt, S. A. (2011). Hemifield effects of spatial attention in early human visual cortex. European Journal of Neuroscience, 33, 2349-2358.

Kraft, A., Müller, N. G., Hagendorf, H., Schira, M. M., Dick, S., Fendrich, R. M., \& Brandt, S. A. (2005). Interactions between task difficulty and hemispheric distribution of attended locations: Implications for the splitting attention debate. Cognitive Brain Research, 24, 19-32.

Kraft, A., Pape, N., Hagendorf, H., Schmidt, S., Naito, A., \& Brandt, S. A. (2007). What determines sustained visual attention? The impact of distracter positions, task difficulty and visual fields compared. Brain Research, 1133, 123-135.

Large, M.-E., Culham, J., Kuchinad, A., Aldcroft, A., \& Vilis, T. (2008). fMRI reveals greater within- than between-hemifield integration in the human lateral occipital cortex. European Journal of Neuroscience, 27, 3299-3309.

Malinowski, P., Fuchs, S., \& Müller, M. M. (2007). Sustained division of spatial attention to multiple locations within one hemifield. Neuroscience Letters, 414, 65-70.

McMains, S. A., \& Somers, D. C. (2004). Multiple spotlights of attentional selection in human visual cortex. Neuron, 42, 677-686.

McMains, S. A., \& Somers, D. C. (2005). Processing efficiency of divided spatial attention mechanisms in human visual cortex. Journal of Neuroscience, 25, 9444-9448.

Morgan, S. T., Hansen, J. C., \& Hillyard, S. A. (1996). Selective attention to stimulus location modulates the steady-state visual evoked potential. Proceedings of the National Academy of Sciences, U.S.A., 93, 4770-4774.

Müller, M. M., \& Hübner, R. (2002). Can the spotlight of attention be shaped like a doughnut? Evidence from steady-state visual evoked potentials. Psychological Science, 13, 119-124.

Müller, M. M., Malinowski, P., Gruber, T., \& Hillyard, S. A. (2003). Sustained division of the attentional spotlight. Nature, 424, 309-312.
Müller, M. M., Picton, T. W., Valdés-Sosa, P., Riera, J., Teder-Sälejärvi, W. A., \& Hillyard, S. A. (1998). Effects of spatial selective attention on the steady-state visual evoked potential in the $20-28 \mathrm{~Hz}$ range. Cognitive Brain Research, 6, 249-261.

Müller, M. M., Teder-Sälejärvi, W. A., \& Hillyard, S. A. (1998). The time course of cortical facilitation during cued shifts of spatial attention. Nature Neuroscience, 1, 631-634.

Müller, N. G., \& Kleinschmidt, A. (2004). The attentional "spotlights" penumbra: Center-surround modulation in striate cortex. NeuroReport, 15, 977-980.

Müller, N. G., Mollenhauer, M., Rösler, A., \& Kleinschmidt, A. (2005). The attentional field has a Mexican hat distribution. Vision Research, 45, 1129-1137.

Murray, S. O. (2008). The effects of spatial attention in early human visual cortex are stimulus independent. Journal of Vision, 8, 1-11.

Pestilli, F., Carrasco, M., Heeger, D. J., \& Gardner, J. L. (2011). Attentional enhancement via selection and pooling of early sensory responses in human visual cortex. Neuron, 72, 832-846.

Peterson, M., \& Juola, J. F. (2000). Evidence for distinct attentional bottlenecks in attention switching and attentional blink tasks. The Journal of General Psychology, 127, 6-26.

Posner, M. I., Snyder, C. R., \& Davidson, B. J. (1980). Attention and the detection of signals. Journal of Experimental Psychology, 109, 160-174.

Purves, D., Augustine, G. J., Fitzpatrick, D., Hall, W. C., LaMantia, A.-S., McNamara, J. O., \& Williams, S. M. (Eds.) (2004).Neuroscience (3rd ed., p, 773). Sunderland, UK: Sinauer Associates.

Quigley, C., Andersen, S. K., \& Müller, M. M. (2012). Keeping focused: Sustained spatial selective visual attention is maintained in healthy old age. Brain Research, 1469, 24-34.

Reeves, A., \& Sperling, G. (1986). Attention gating in short-term visual memory. Psychological Review, 93, 180-206.

Regan, D. (1989). Human brain electrophysiology: Evoked potentials and evoked magnetic fields in science and medicine (p. 672). New York: Elsevier.

Reynolds, J. H., \& Heeger, D. J. (2009). The normalization model of attention. Neuron, 61, 168-185.

Schwartz, S., Vuilleumier, P., Hutton, C., Maravita, A., Dolan, R. J., \& Driver, J. (2005). Attentional load and sensory competition in human vision: Modulation of fMRI responses by load at fixation during task-irrelevant stimulation in the peripheral visual field. Cerebral Cortex, 15, 770-786.

Sereno, A. B., \& Kosslyn, S. M. (1991). Discrimination within and between hemifields: A new constraint on theories of attention. Neuropsychologia, 29, 659-675.

Störmer, V. S., Alvarez, G. A., \& Cavanagh, P. (2014). Within-hemifield competition in early visual areas limits the ability to track multiple objects with attention. Journal of Neuroscience, 34, 11526-11533.

Störmer, V. S., Winther, G. N., Li, S.-C., \& Andersen, S. K. (2013). Sustained multifocal attentional enhancement of stimulus processing in early visual areas predicts tracking performance. Journal of Neuroscience, 33, 5346-5351.

Sundberg, K. A., Mitchell, J. F., \& Reynolds, J. H. (2009). Spatial attention modulates center-surround interactions in macaque visual area V4. Neuron, 61, 952-963.

Walter, S., Quigley, C., \& Müller, M. M. (2014). Competitive interactions of attentional resources in early visual cortex during sustained visuospatial attention within or between visual hemifields: Evidence for the different-hemifield advantage. Journal of Cognitive Neuroscience, 26, 938-954.

Weichselgartner, E., \& Sperling, G. (1987). Dynamics of automatic and controlled visual attention. Science, 238, 778-780. 